\title{
Modification and initial testing of the Family Communication Questionnaire (FCQ) for Japanese child-rearing families in the period one month postpartum
}

\author{
Tae Kawahara ${ }^{1}$, Kanako Matsueda ${ }^{2}$, Maiko Yasuzato ${ }^{1}$, Akemi Yamazaki*1 \\ ${ }^{1}$ Division of Health Sciences, Area of Nursing Science, Graduate School of Medicine, Osaka University, Japan \\ ${ }^{2}$ Department of Nursing, The Faculty of Health Science, Kyoto Koka Women's University, Japan
}

Received: October 7, 2018

Accepted: January 23, 2019

Online Published: January 29, 2019

DOI: $10.5430 /$ jnep.v9n6p7

URL: https://doi.org/10.5430/jnep.v9n6p7

\begin{abstract}
The aim of the present study was to develop and validate a modified version of the Family Communication Questionnaire (FCQ) for women in the period one month postpartum with a focus on communication with the woman's partner, own mother, and friends. The FCQ can measure the frequency of communication between women and their partners, own mothers, and friends across four childcare-related domains by methods such as face-to-face interaction, telephone, e-mail, and social networking services. In the present study, a total of 500 mothers were recruited from a facility in the Kansai region of Japan from February to July 2017 to complete a survey including a modified version of the FCQ and the General Functioning Subscale of the Family Assessment Device (FAD-GF). Two weeks later, 49 of 100 mothers asked completed a test-retest. After excluding responses that were incomplete for $>10 \%$ of the items in each domain, 399 (79.8\%) valid responses were included in the analysis. The distribution of scores showed a floor effect for all scores except for those with the husband. The intraclass correlation coefficients for couple (woman-her partner), woman-her own mother, and woman-her friends were 0.719-0.897, 0.871-0.987, and -0.088-0.452, respectively, and Cronbach's $\alpha$ coefficients were 0.886, 0.897, and 0.897, respectively. The FCQ and FAD-GF total scores were negatively correlated. The modified FCQ developed in the present study shows good internal consistency, stability, and validity for communication between mothers, their husbands, and their own mothers during the period one month postpartum.
\end{abstract}

Key Words: Communication, Family, Childcare, Mother

\section{INTRODUCTION}

Women are most susceptible to developing postpartum depression (PPD) in the period one month postpartum. However, maladjustment to childcare, including depression, is low when women, together with their partners, receive support and information from fellow parents living in their community. ${ }^{[1-5]}$ The incidence of PPD is low when little stress exists in the current marital relationship, even if the woman has a history of depression. ${ }^{[6]}$ Particularly in Japan, many women return to their parent's home for childbirth (a phenomenon referred to as "satogaeri"), and women who have friends with whom they can discuss childcare score low on the Edinburgh Postnatal Depression Scale at one month postpartum. ${ }^{[7]}$ This suggests that is possible to summarize PPD in the context of

*Correspondence: Akemi Yamazaki; Email: akemiyamazaki@ sahs.med.osaka-u.ac.jp; Address: 1-7 Yamadaoka, Suita, Osaka 565-0871 Japan. 
internal and external family communication, meaning that a woman can maintain her health while caring for an infant if functional communication between the woman and her partner and own mother exists within the family, as well as between the woman and fellow parents outside of the family.

In recent years, particularly because of the spread of smartphones, the methods by which people communicate have changed greatly. ${ }^{[8]}$ Intra-family communication utilizing real-time photos and videos that do not require meeting directly has become possible between family, friends, and others. It is expected that Japanese child-rearing families use a variety of tools for internal/external communication. However, the communication tools used by women in the period one month postpartum, whose communication methods can differ remarkably depending on where they live after giving birth, has yet to be clarified. Therefore, the purpose of the present study was to develop and validate a modified version of the Family Communication Questionnaire (FCQ) for women in the period one month postpartum with a focus on the mother's communication with her partner, own mother, and friends.

\subsection{Conceptual framework}

According to the family systems theory, a family is a living unit that grows while changing and interacting with other systems inside and outside of the family unit. In family development theory, the period one month postpartum is perceived as a transitional period in which a young couple procreates and raises a family while receiving support and influence from the family in which they were born and raised. ${ }^{[9]}$ Families are primarily made up of husband-wife, mother-child, and father-child subsystems, and a single family unit raises infants while communicating with grandparents and other close relatives. The family unit also communicates with friends outside of the family who constitute a source of social support in relation to childcare. We therefore operationalized the objects of communication in our instrument, a modified version of the FCQ, so that partners and biological mothers were included inside the family unit, while friends were included outside of the family unit.

The content of family communication during the period one month postpartum may include the matter of the child him or herself, requests for support with childcare, sharing feelings such as anxiety over childcare, and exchanging information on childcare. Family theory states that, in general, functional communication observed in healthy families involves the transmitter and receiver of information listening carefully and providing feedback while confirming that their communication partner has correctly understood the clear and concise message being conveyed. ${ }^{[10]}$ However, this presupposes mainly face-to-face communication. The type of information communicated and the communication methods used between families during the period one month postpartum needs to be clarified. We therefore examined these matters in a preliminary survey, as detailed below.

\subsection{Development of the study instrument}

Ishii-Kuntz, who is a family sociologist, developed the original FCQ as an instrument to measure the frequency and method (face-to-face, phone, e-mail) of communication among parents raising pre-kindergarten children. ${ }^{[11]}$ Each domain had the same items: "obtaining information necessary for childcare", "talking about problems related to childcare", "contacting childcare provider", and "showing pictures and videos of the child". Parents rated their frequency of communication for each item as follows: not: 1 ; 1-2 times/month: 2; 1 time/week: 3; 2-3 times/week: 4; 1-5 times/day: 5; and more than 5 times/day: 6 . Higher scores mean more frequent family communication. She engaged in international comparative research using this instrument to assess the positive aspects of family communication using information technology (IT), primarily in relation to the participation of fathers in the care of school-age children. Ishii-Kuntz notes that families who actively make use of IT tools to communicate have a higher degree of satisfaction with family communication.

First, we obtained permission from Ishii-Kuntz to modify the FCQ in order to investigate the topics and methods of family communication among women in the period one month postpartum. Next, from October to November 2016, we conducted semi-structured interviews with six women-their own mother pairs, three couple (women and their partner) pairs, and one woman-her mother-in-law pair. Interviews lasted 27-43 min (mean duration, $33.9 \mathrm{~min}$ ). The mean ( \pm standard deviation [SD]) age of the women in the period one month postpartum was $33.3 \pm 3.5 \mathrm{y}$. Data were transcribed verbatim and analyzed qualitatively to find out who the participants communicated with, how often they communicated, what they communicated about, and what communication methods they used. The views of colleagues and two midwives were also collected and examined.

Through the interviews, it was revealed that in addition to the face-to-face, phone, and e-mail domains formulated by Ishii-Kunz, social networking services (SNS) were an important tool for communication with specific and nonspecific people. Therefore, we adopted four domains as subscales of the modified FCQ. Almost all communication topics consisted of the original FCQ contents, except for 'contacting childcare provider (bathing, shopping, pick-up, etc.), which we changed to 'contacting childcare provider (pick-up, dropoff, playing, etc.)' in accordance with the period one month 
postpartum.

The feasibility of applying the results was investigated through a preliminary test involving men and women with childcare experience and 10 nursing researchers working in the field of mother and child nursing. Based on the above, the various recent methods of communication were given descriptions so that they were comprehensible to the participants, the content of the modified FCQ was finalized, and a survey was conducted. The methods of communication were as follows: "face-to-face: communication through direct meetings", "phone: communication using a cell phone or Skype", "e-mail: communication through individual emails", and "SNS: communication within a specific group using a messaging application (such as Line or WhatsApp Messenger)".

\section{METHOD}

\subsection{Participants}

Women in the period one month postpartum who were visiting a single obstetrical and gynecological hospital in the urban area of the Kansai region for a medical checkup and met the inclusion criteria were recruited. The exclusion criteria were: became pregnant at $<20$ y of age; gave birth at less than 37 weeks gestation; and unable to rooming-in because of maternal or neonatal transport. If responses were incomplete for $>10 \%$ of the items in each domain, the mother was excluded from the analysis. In total, 500 participants were finally recruited from the facility from February to July 2017.

\subsection{Measures}

The FCQ included items on the participants' demographic characteristics, including age, medical history, educational background, birth method, birth week and destination after discharge. The contents of the survey form included the participants' attributes, the FCQ, and the General Functioning Subscale of the Family Assessment Device (FAD-GF) to verify the criterion-related validity. Among all participants, 100 were asked to complete a test-retest 2 weeks later.

The FAD-GF, which was designed to evaluate families according to the McMaster Model of Family Functioning, ${ }^{[12]}$ was used to check concurrent validity. The FAD is composed of seven scales. Among these, the FAD1 survey is for families in which the participant was born and raised, while the FAD2 survey is for the participant's current family. Higher scores on the FAD-GF indicate worse family function.

\subsection{Ethical considerations}

All study procedures were approved by the Institutional Review Board of Osaka University (IRB: 16194-4). All participants received an oral explanation of the study contents and protocol and provided written informed consent to participate. All participant data were digitized and anonymized to ensure confidentiality.

\subsection{Statistical analyses}

Statistical analyses were performed using SPSS for Windows (version 22; IBM, Tokyo, Japan). Descriptive statistics were used to summarize the participants' demographic characteristics, and the means, SDs, and medians were calculated. In addition, 2-week test-retest reliability was assessed using intraclass correlation coefficients (ICCs) and internal consistency was measured using Cronbach's $\alpha$. Construct validity was examined by calculating Pearson's correlation coefficient $(r)$ between the FCQ and FAD-GF. After examining whether the population was normally distributed using the Kolmogorov-Smirnov test, a nonparametric Mann-Whitney $\mathrm{U}$ test was used to compare the two groups. $p$ values $\leq 0.05$ were considered statistically significant.

\section{RESULTS}

\subsection{Participants}

In total, 500 mothers were analyzed (mean age \pm SD, 34.2 $\pm 4.4 \mathrm{y}$; age range, 20-44 y; mean age \pm SD of fathers, 36.2 $\pm 5.6 \mathrm{y}$; age range, 22-58 y). After excluding responses that were incomplete for $>10 \%$ of the items in each domain, the number of valid responses was $399(79.8 \%)$. Of these, $206(51.6 \%)$ were primiparas and $193(48.4 \%)$ were multiparas. The mean birth week at delivery was $39.0 \pm 1.1$ and the mean birth weight was 3,048.0 $\pm 366.0 \mathrm{~g}$. In total, $30(7.5 \%)$ respondents had a history of mental or physical illness, and 221 (55.4\%) had a university-level college education. Regarding the destination at discharge, 188 (47.1\%) respondents indicated the maternal parent's home and 195 (48.9\%) the couple's own home (see Table 1).

\subsection{FCQ score distribution}

Only about $40 \%$ of the women communicated face-to-face with their husband on a daily basis. The next most commonly used tool for communication with husbands was e-mail, with $40 \%$ "showing pictures and videos of the child" to their husband. About $60 \%$ of the participants contacted their own mother about childcare ("contacting childcare provider") every day, and more than half of the participants contacted their own mother on a daily basis ("talking about problems related to childcare"). The next most common tool for communication with own mothers was e-mail; approximately half of the participants used e-mail for "showing pictures and videos of the child". Participants also exchanged many e-mails with friends, with $40 \%$ "showing pictures and videos of the child", "talking about problems related to childcare", and "obtaining information necessary for childcare" once a 
week. A histogram of the total scores for face-to-face, phone, and e-mail communication with the husband, face-to-face communication with the own mother, and e-mail communication with friends showed a normal distribution. However, a floor effect was seen for other scores (see Table 2).

Table 1. Characteristics of the participants $(\mathrm{n}=399)$

\begin{tabular}{ll}
\hline Characteristics & N (\%) \\
\hline Age, mean (SD), $\mathbf{y}$ & \\
$\quad$ Answer (puerpera) & $34.2(4.4)$ \\
$\quad$ Husband & $36.2(5.6)$ \\
Type of puerpera, n (\%) & \\
$\quad$ Primipara & $206(51.6)$ \\
$\quad$ Multipara & $193(48.4)$ \\
Birth method, n (\%) & \\
$\quad$ Normal delivery & $267(66.9)$ \\
Cesarean section & $107(33.1)$ \\
Number of birth weeks, week (SD) & $39.0(1.1)$ \\
$\quad$ Baby's birth weight, g (SD) & $3,048.0(366.0)$ \\
Medical history, n (\%) & \\
$\quad$ Yes & $30(7.5)$ \\
$\quad$ No & $369(92.5)$ \\
Educational background, n (\%) & \\
Junior and senior high school & $28(7.0)$ \\
Vocational school & $61(15.3)$ \\
Junior college & $55(13.8)$ \\
University or college & $221(55.4)$ \\
Discharge destination & $188(47.1)$ \\
Maternal parent's house & $195(48.9)$ \\
Couple's own house & $13(3.3)$ \\
Husband's parental house & $1(0.3)$ \\
Other &
\end{tabular}

\subsection{Reliability}

\section{Test-Retest reliability and internal consistency}

We asked 100 of the mothers to take part in a test-retest 2 weeks after the survey, 49 of whom agreed. The ICCs for couple (woman-her partner), woman-her own mother, and woman-her friends were $0.719-0.897,0.871-0.987$, and $-0.088-0.452$, respectively, and Cronbach's $\alpha$ coefficients were $0.886,0.897$, and 0.897 , respectively (see Table 3 ).
Table 2. Family Communication Questionnaire (FCQ) score distribution

\begin{tabular}{lll}
\hline Items & & Mean \pm SD \\
\hline \multirow{4}{*}{ Couple } & Face-to-face & $14.9 \pm 5.7$ \\
& Phone & $11.1 \pm 6.0$ \\
& E-mail & $13.3 \pm 5.9$ \\
& SNS & $7.3 \pm 5.2$ \\
Woman-her own mother & Face-to-face & $17.0 \pm 6.7$ \\
& Phone & $7.5 \pm 5.3$ \\
& E-mail & $8.2 \pm 5.5$ \\
& SNS & $5.7 \pm 4.3$ \\
& Face-to-face & $6.3 \pm 3.0$ \\
Woman-her friends & Phone & $5.2 \pm 2.7$ \\
& E-mail & $8.8 \pm 4.1$ \\
& SNS & $6.7 \pm 3.9$ \\
\hline
\end{tabular}

\subsection{Validity}

\subsubsection{Concurrent validity}

Next, to assess concurrent validity, we examined whether the FCQ and FAD-GF total scores were negatively correlated. Since each target was explained by a different version of the FAD-GF, communication between couples was calculated using the FAD-GF 2, and that between woman-her own mother and woman-her friends using the FAD-GF 1. Strong negative correlations were seen between couple's FCQ and FAD-GF 2 and between woman-her own mother's FCQ and FAD-GF $1(r=-.316$ and -.284 , respectively), and a moderate negative correlation was observed between woman-her friend's FCQ and FAD-GF $1(r=-.139)$ (see Table 3).

\subsubsection{Known-groups validity}

In the present study, we had the following three hypotheses. Hypothesis 1: Women who were discharged to their own home would have higher scores in the couple face-to-face domain compared with those discharged to their maternal parents' home. Hypothesis 2: Women who were discharged to their maternal parents' home (satogaeri) would have higher scores in the woman-her own mother face-to-face domain compared with those discharged to their own home. Hypothesis 3: Younger women (aged $>34$ y) would have higher scores in the woman-her friend SNS domain compared with older women (aged $>35 \mathrm{y})$.

Table 3. Test-retest correlations, internal consistency, and correlation of FCQ and FAD-GF

\begin{tabular}{|c|c|c|c|c|c|c|}
\hline \multirow{2}{*}{ Communication } & \multirow{2}{*}{$\begin{array}{l}\text { Sample } \\
\text { size, } \mathbf{n}\end{array}$} & \multicolumn{2}{|c|}{$95 \%$ confidence revel } & \multirow{2}{*}{ Cronbach's $\alpha$} & \multicolumn{2}{|c|}{ FAD-GF, correlation $r$} \\
\hline & & Minimum & Maximum & & FAD-GF 1 & FAD-GF 2 \\
\hline Couple & 399 & .719 & .897 & 0.886 & & $-.316 * *$ \\
\hline Woman-her own mother & 395 & .871 & .987 & 0.897 & $-.284^{* *}$ & \\
\hline Woman-her friends & 399 & -.088 & .452 & 0.897 & $-.139 * *$ & \\
\hline
\end{tabular}

$* * p<.01$ 
All three domains showed a non-parametric distribution using the Kolmogorov-Smirnov test of normality $(p<.01)$. Table 3 shows the medians and z-scores of the two groups. Women who were discharged to their own home (median, 18.00; interquartile range [IQR], 13.00-20.00) showed significantly higher scores in the couple face-to-face communication domain compared with women who were discharged to their maternal parents' home (median, 12.00; IQR, 8.00$16.00)(\mathrm{z}=-6.662, p<.01)$. Conversely, women who were discharged to their maternal parent's home (median, 20.00; IQR, 17.00-24.00) showed significantly higher scores in the woman-her own mother face-to-face communication domain compared with women who were discharged to the couple's own home (median, 15.00; IQR, 7.25-20.00) $(\mathrm{z}=-8.447, p$ $<.01)$. Finally, younger women showed significantly higher scores (median, 6.00; IQR, 4.00-6.00) in the woman-her friends SNS domain compared with older women (median, 4.00; IQR, 4.00-8.00) $(\mathrm{z}=-3.110, p<.01)$. (see Table 4).

Table 4. Known-groups validity

\begin{tabular}{|c|c|c|c|c|}
\hline Subscale & Subgroups & Sample size, $n$ & Score median (IQR) & z score \\
\hline \multicolumn{5}{|c|}{ Couple face-to-face communication domain } \\
\hline & Couple’s own house & 188 & $18.00(13.00-20.00)$ & \multirow[t]{2}{*}{$-6.662 * *$} \\
\hline & Maternal parent's house & 195 & $12.00(8.00-16.00)$ & \\
\hline \multicolumn{5}{|c|}{ Woman-her own mother face-to-face communication domain } \\
\hline & Couple’s own house & 184 & $15.00(7.25-20.00)$ & \multirow[t]{2}{*}{$-8.447 * *$} \\
\hline & Maternal parent's house & 195 & $20.00(17.00-24.00)$ & \\
\hline \multicolumn{5}{|c|}{ Woman-her friends SNS domain } \\
\hline & Younger group & 196 & $6.00(4.00-6.00)$ & \multirow[t]{2}{*}{$-3.110 * *$} \\
\hline & Elder group & 203 & $4.00(4.00-8.00)$ & \\
\hline
\end{tabular}

$* * p<.01$

\section{Discussion}

Family functioning scales such as the FAD and Family Adaptability and Cohesion Evaluation Scales (FACES) focus on communication as their subordinate concept. In these instruments, clear, frank, and direct family communication is defined as "healthy" and measured accordingly. ${ }^{[12,13]}$ However, communication in Japanese families places emphasis on nonverbal communication among other characteristics, ${ }^{[14]}$ and a healthy style of communication regarded by Western family functioning scales is not always preferable, which causes considerable difficulty with measurement. The present instrument, the FCQ, emphasizes measurement of the frequency of family communication in its four domains related to childcare.

The distribution of scores for intra-family communication showed a floor effect for all scores apart from those for communication with the husband (face-to-face, phone, and e-mail) and own mother (face-to-face). Despite a certain number of participants who communicated with their own mother by phone and e-mail and with their husband by SNS, the majority of the participants did not use these tools for communication during the period one month postpartum. However, at the same time, half of the participants returned to their parent's home for childbirth, and married couples not only used face-to-face communication, but also had recently

Published by Sciedu Press adopted non-face-to-face communication such as phone and e-mail in the period one month postpartum.

In recent years, a number of reports have been published on how mothers of infants are making use of communication in a digital society, in addition to face-to-face social support networks, in their parenting. ${ }^{[15,16]}$ Studies of women in the postpartum period making use of SNS tools as a means of social support to seek out information and obtain advice have also been reported. ${ }^{[17,18]}$ However, despite the fact that the participants in the present study were found to communicate with their friends via e-mail in the period one month postpartum, with the exception of e-mail, the distribution of scores for extra-family communication revealed low scores and a floor effect for face-to-face and phone communication with friends. Moreover, in a subsequent test-retest, stability was achieved for all items except communication with friends. The reason for this is that in Japan, first-time mothers are particularly unlikely to leave their home with their child before the one month postpartum checkup, suggesting that extra-family communication differs before and after this period.

The original version of the FCQ is used to assess communication between mothers and fathers of school-age children. Ishii-Kuntz has pointed out that child-rearing families who 
have used IT and social media skillfully in recent years engage in frequent family communication. In the present study, not only communication with partners, but also that with participants' own mothers and friends was measured, because women's own mothers play a key role in Japanese families raising children during the period one month postpartum, and because maintaining communication with fellow parents is essential for parous women. However, few participants in the present study used tools other than face-to-face methods to communicate with their own mother, and e-mail to communicate with friends. Smartphones are used by $90 \%$ of pregnant and parturient women in low-income groups, regardless of race or ethnicity, ${ }^{[19]}$ and according to a FY2017 White Paper on Information and Communications in Japan published by the Ministry of Internal Affairs and Communications, approximately $90 \%$ of Japanese people in their 20s and 30s use cellular or smartphones. ${ }^{[8]}$ This trend is likely to accelerate in the future. The women in the present survey were relatively older, with a mean age of $34.3 \pm 4.8 \mathrm{y}$. A future survey conducted on a younger age group might reveal greater numbers of people using phones, e-mail, and SNS to communicate, as would a survey of women's own mothers, who represent a generation gradually familiarizing themselves with communication via cellular and smartphones. Furthermore, a survey conducted on mothers caring for infants throughout the entire period of infancy (1-12 months of age) could be expected to reveal a variety of means of communication with friends.

Despite the aforementioned issues, participants in the period one month postpartum were guaranteed internal consistency and stability in their communication with their husbands and own mothers. Known-groups validity was also guaranteed; participants with high FAD scores for the family they were building with their husbands had high FCQ scores for their husbands, and those with high FAD scores for their parent's home had high FCQ scores for their own mother.

\section{NURSING IMPLICATIONS AND CONCLU- SION}

In conclusion, in the present study, we modified an instrument that measures IT-based communication in families with school-age children and developed an instrument for 399 women during the period one month postpartum, the FCQ. Our tool is capable of measuring the frequency of communication between women and their partners, own mothers, and friends by methods such face-to-face, phone, e-mail and SNS communication, across four domains related to childcare. During the period one month postpartum, an undeniable limit existed in measurements of communication with friends. Assessing communication among younger generations and during their babies' entire period of infancy remains a future challenge. However, the modified FCQ developed in the present study showed good internal consistency, stability, and validity for communication between mothers and their husbands and own mothers during the period one month postpartum.

\section{ACKNOWLEDGEMENTS}

The authors are grateful to the study participants. The present study was supported by a Grant-in-Aid for Scientific Research KAKENHI Challenging Exploratory Research (16K15925).

\section{Authors' CONTRIBUTIONS}

Tae Kawahara and Kanako Matsueda contributed equally to this work.

\section{CONFLICTS OF INTEREST DisClOSURE}

The authors declare that there is no conflict of interest.

\section{REFERENCES}

[1] Beck C. A meta-analysis of predictors of postpartum depression. Nursing Research. 1996; 45(5): 297-303. https ://doi .org/10.1 097/00006199-199609000-00008

[2] Beck C. A meta-analysis of the relationship between postpartum depression and infant temperament. Nursing Research. 1996b; 45(4) 225-230. PMid:8700656 https://doi.org/10.1097/00006199 $-199607000-00006$

[3] Beck C. Postpartum depressed mothers' experiences interacting with their children. Nursing Research. 1996c; 45(2): 98104. PMid:8604372 https://doi.org/10.1097/00006199-1 99603000-00008

[4] Beck C. Predictors of postpartum depression-An update. Nursing Research. 2001; 50(5): 275-285. https://doi .org/10.1097/00
006199-200109000-00004

[5] Maehara K, Mori E, Tsuchiya M, et al. Factors affecting maternal confidence among older and younger Japanese primiparae at one month post-partum. Japan Journal of Nursing Science. 2016; 13(4): 424-436. PMid:27170452 https://doi .org/10.1111/jjns. 12123

[6] Banker J, LaCoursiere D. Postpartum depression: Risks, protective factors, and the couple's relationship. Issues in Mental Health Nursing. 2014; 35(7): 503-508. PMid:24963850 https://doi .org/10 $.3109 / 01612840.2014 .888603$

[7] Takahashi Y, Tamakoshi K. Factors associated with early postpartum maternity blues and depression tendency among Japanese mothers with full-term healthy infants. Nagoya Journal of Medical Sciences. 2014; 76: 129-138. PMid:25129999

[8] Ministry of Internal Affairs and Communications. White paper infor- 
mation and communications in Japan. Chapter 1. Present and future of smartphone economy. 2017. Available from: http://www. soum u.go.jp/

[9] Calvin K, Bylund C, Brommel B. Family communication cohesion and change. 6th ed. Boston, MA: Pearson Education. 2004.

[10] Friedman M, Bowden V, Jones E. Family nursing research, theory, \& practice (5th ed.). Upper Saddle River, NJ: Prentice Hall. 2003.

[11] Ishii-Kuntz M. The use of Internet technology and family relationships in information society: Japan-US comparison. Grant-in-Aid for Scientific Research paper. 2012.

[12] Epstein NB, Baldwin DS, Bishop DS. The McMaster Family Assessment Device. Journal of Marital and Family Therapy. 1983; 9: 171-180. https://doi.org/10.1111/j.1752-0606.1983.tb0 1497.x

[13] Olson DH. Commentary: three-dimensional (3-D) Circumplex Model and revised scoring of FACESIII. Family Process. 1991; 30: 74-79. https://doi.org/10.1111/j.1545-5300.1991.00074.x

[14] McDaniel E. Japanese nonverbal communication: A reflection of cultural themes. In L. Samovar, R. Porter, \& E. McDaniel (Eds.), Intercultural communication: A reader. 14th ed. Boston, MA: Wadsworth; 2015.
[15] Lupton D, Pedersen S. An Australian survey of women's use of pregnancy and parenting apps. Women and Birth. 2016; 29(4): 368-375. PMid:26874938 https://doi .org/10.1016/j . wombi . 2016.0 1.008

[16] Lupton D, Pedersen S, Thomas GM. Parenting and digital media: From the early Web to contemporary digital society. Sociology Compass. 2016; 10(8): 730-743. https://doi.org/10.1111/soc4.1 2398

[17] Jang J, Dworkin J, Hessel H. Mothers' use of information and communication technologies for information seeking. Cyberpsychology, Behavior, and Social Networking. 2015; 18(4): 221-227. PMid:25803204 https://doi.org/10.1089/cyber. 2014.053 3

[18] Morris RM. Social networking site use by mothers of young children In Proceedings of the 17th ACM conference on computer supported cooperative work and social computing (pp. 1272-1282). ACM. Baltimore, MD; United States. 2014; 15-19 February.

[19] Chilukuri N, West M, Henderson J, et al. Information and communication technology use among low-income pregnant and postpartum women by race and ethnicity: A cross-sectional study. Journal of Medical Internet Research. 2015; 17(7): e163. 